\title{
Identificación Organizacional y Satisfacción Laboral: Diferencia entre Empresas Públicas y Privadas
}

\author{
Organizational Identification and Job Satisfaction: Difference between Public and \\ Private Business
}

Ana Álvarez Muelas, Andreea Ionela Dinu, Irene Marín Segura, Teresa Marrero Marrero, Laura Mas Cuesta y Marina Muñoz González. Universidad de Granada

\author{
Artículo dirigido por: Inmaculada Valor Segura. Departamento de Psicología Social. Universidad de Granada \\ Fecha de recepción: 19 de marzo de 2014. \\ Fecha de revisión: 19 de marzo de 2014. \\ Fecha de aceptación: 11 de abril de 2014.
}

\section{Resumen}

En el presente estudio el objetivo es comparar la satisfacción laboral y la identificación organizacional entre los empleados de empresas públicas y privadas. Para ello se ha contado con una muestra de 95 adultos. Se partió de la siguiente hipótesis: la satisfacción laboral y la identificación organizacional están correlacionadas positivamente, asimismo las empresas privadas tendrán una mayor satisfacción laboral e identificación organizacional que las empresas públicas. Tras el análisis de los resultados se observa una correlación entre la satisfacción laboral y la identificación organizacional siendo la primera mayor en las empresas privadas que en las públicas. Sin embargo, no se han obtenido resultados significativos relacionados con la identificación organizacional.

Palabras claves: Identificación organizacional, satisfacción laboral, empresa

\begin{abstract}
In the present study our objective is to compare job satisfaction and organizational identification between workers belonging to both public and private businesses. To achieve this target we used two evaluation instruments: "Cuestionario de Satisfacción Laboral S10/12 y la escala de Identificación Grupal (Tarrant, 2002)"which were passed to a group of 95 adults. These were our hypothesis: job satisfaction and organizational identification are positively correlated, private bussineses will have a bigger job satisfaction and organizational identification than public businesses. After analizing our results we observed a positive correlation between job satisfaction and organizational identification. The results also proved the existence of a bigger job satisfaction in private businesses in comparison to public businesses. We have not obtained any significant results referring to organizational identification.
\end{abstract}

Keywords: Organizational identification, job satisfaction, business

\section{Introducción}

En 1943 Abraham Maslow ya propuso dentro de su Pirámide de Necesidades la necesidad de pertenencia a un grupo, requisito indispensable para poder llegar a la autorrealización personal. Según la Teoría de la Identificación Social de Tajfel (1982) la identidad social (intergrupal) es el conocimiento individual de que uno pertenece a ciertos grupos sociales junto con algunas significaciones emocionales y valorativas que van unidas al grupo. Se distingue de la identidad personal porque no se basa en 
atributos que se refieren al aspecto físico, cualidades intelectuales entre otros rasgos personales. Por tanto la persona se identifica dentro de un continuo donde en un extremo se sitúa la identificación personal y en el otro extremo la identificación social. Una mayor identificación con uno de estos extremos debilita la identificación del otro, es decir, a mayor identidad personal menor identidad social y viceversa. La despersonalización (Turner, 1982) resulta de la identificación con el extremo social, en esta situación la persona se deja de percibir como un ente individual y pasa a ser un ente grupal, intercambiable por cualquier otro individuo del mismo grupo.

Identidad organizacional

Henry, Arrow y Carini (1999) contemplan tres diferencias entre identidad social e identificación grupal (organizacional). Primero, la identificación grupal incluye a grupos interactivos a diferencia de la identificación social que contempla el grupo "en mente". La segunda diferencia es que la identificación grupal se refiere a procesos intragrupales y la social a procesos intergrupales. Por último la identidad social es una variable dicotómica (ser o no miembro de un grupo social) (Turner, 1982) mientras que la identificación grupal es medida como una variable continua (Darran, 2002; Hinkle, Taylor, Fox-Cardamone y Crook, 1989).

La identificación organizacional presenta cuatro características clave. En primer lugar, al ser una categorización de la información social y no formar necesariamente parte de una conducta se considera como una percepción en la que el individuo se percibe a sí mismo psicológicamente enlazado con el grupo. La conducta puede darse a partir de esta identificación. La segunda característica es que el individuo es capaz de experimentar el éxito y el fracaso del grupo. En tercer lugar, la identificación no significa internalización de valores sino identificación con los mismos. Por último, las personas suelen describirse a sí mismas con el concepto de pertenencia a un grupo haciendo referencia a su realidad social en la coexistencia con otros grupos.

Existen ciertos factores que hacen más favorable la identificación con un cierto grupo como puede ser la distintividad (saliencia del grupo), prestigio del grupo, saliencia de los exogrupos (percepción de homogeneidad dentro del propio grupo y hetereogeneidad hacia los otros grupos) y demás factores asociados como la semejanza, la proximidad física y la vinculación, que fomentan la formación de un grupo (Ashforth y Mael, 1989).

Daan van Knipenberg y van Schie (2000) estudiaron la existencia de factores que afectan a la identificación con la organización. Entre ellos encontramos el estatus, tamaño, semejanza y contexto. Con respecto al estatus, se prefieren organizaciones con un elevado nivel de este ya que se consigue crear una imagen positiva del grupo. El tamaño relativo es importante en la identificación ya que los grupos pequeños fomentan tanto la identificación individual como la pertenencia al grupo. Sin embargo los de gran tamaño hacen que la distintividad indiviual quede menguada. La semejanza se refiere a que a mayor similitud percibida con el grupo, mayor identificación con el mismo. Por último, la identificación es dependiente del contexto.

Tradicionalmente se ha hablado de identificación grupal como una variable unidimensional. Actualmente Cava, Buelga, Herrero, y Musitu (2011) han distinguido tres dimensiones dentro de esta escala: autocategorización, valoración grupal y compromiso grupal, acercándose mucho más a la realidad de este concepto y consiguiendo evaluar la identificación organizacional en consonancia con los planteamientos teóricos de la Teoría de la Identidad social. 


\section{Satisfacción laboral}

Uno de los modelos teóricos más difundidos para explicar la satisfacción laboral es el modelo de la discrepancia, el cual defiende la satisfacción como producto de un desequilibrio debido a la comparación del trabajo actual frente al ideal (Locke (1969)).

Según Seashore, y Taver (1975), existen tres puntos de vista prácticos para definir el significado de satisfacción laboral. El primero declara que la satisfacción laboral es un importante pilar de la sociedad. En segundo lugar la satisfacción laboral es un indicador de cómo evolucionará una organización. Si esta se mantiene constante desde sus inicios se puede predecir un correcto funcionamiento de la misma. Por último la satisfacción es un predictor del comportamiento organizativo y también puede servir como referente para planes futuros.

Robbins y Coulter (2000) definen la satisfacción laboral como "una actitud general del empleado frente a su respectivo trabajo". Por otra parte Mottaz (1988) se refiere a la satisfacción laboral como una respuesta emocional como resultado de la medida del estado laboral. En tercer lugar Díez de Castro, García del Junco, Martín Jiménez, y Periáñez Cristóbal (2001) proponen que la satisfacción es un "sentimiento individual que, en términos positivos o negativos, experimentan los individuos en el trascurso de su pertenencia a la organización cuando comparan las recompensas que reciben (tanto extrínsecas como intrínsecas) con las que estiman deberían recibir e, incluso, con aquellas que les gustaría obtener como compensación por los esfuerzos que realizan a favor de la organización".

Estudios recientes como el de Mañas, Salvador, González y Agulló (2007) han estudiado la repercusión que la satisfacción laboral tiene sobre el compromiso en la empresa, o más concretamente sobre la faceta emocional de este: la identificación organizacional. De las distintas variables que pueden influir, estos autores defienden que la satisfacción laboral es la que en mayor medida pronostica el compromiso o identificación de los empleados. Asimismo, se considera vital que las empresas busquen involucrar a sus trabajadores en el desarrollo de sus prácticas de responsabilidad social ya que esto puede a su vez aumentar el sentimiento de pertenencia con la empresa u organización. De esta forma, las organizaciones no solo actúan conforme a las expectativas de responsabilidad social que tiene la comunidad sobre ellos, incluyendo sus propios trabajadores, sino que es más factible que aumente el compromiso y esfuerzo de los mismos empleados como consecuencia de su elevado nivel de satisfacción con su trabajo y con la organización (Gabriunas, 2010).

A partir de toda esta trayectoria teórica planteamos como objetivo de esta investigación comparar las discrepancias entre el nivel de identificación y satisfacción en las empresas privadas y públicas. Por lo tanto, las hipótesis principales de las que partimos serán que una mayor identificación con la organización conllevará a más mayor satisfacción laboral, que la identificación organizacional va a ser mayor en empresas privadas que en públicas y que, por tanto, la satisfacción también será mayor en las empresas privadas que en las públicas.

\section{Método}

\section{Participantes}

La muestra se compone de 95 participantes adultos, todos ellos mayores de 18 años. Se trata de un estudio correlacional intrasujeto, cuya selección de sujetos fue de 
manera incidental ya que se requería que todos estuvieran activos laboralmente. Los sujetos se dividieron en dos grupos en función de si trabajaban en una organización pública o privada (45 sujetos pertenecientes a empresa pública y 50 sujetos a empresa privada)

Instrumentos

Antes de pasar a la aplicación de los cuestionarios, los participantes indicaban su edad, sexo y el tipo de organización en la que trabajan actualmente (pública/privada).

Uno de los instrumentos que se utilizó para la recogida de información fue el Cuestionario de Satisfacción Laboral S10/12 (Meliá y Peiró, 1998). Conéste se pretende medir el nivel de satisfacción que experimentan los sujetos al trabajar en esa organización. Para ello, consta de 12 ítems que evalúa la satisfacción mediante una escala de tipo Likert que abarca 7 puntuaciones desde muy insatisfecho a muy satisfecho (1=muy insatisfecho; 2=bastante insatisfecho; 3=algo insatisfecho; 4=indiferente; $5=$ =algo satisfecho; $6=$ =bastante satisfecho; $7=$ muy satisfecho). La escala muestra un valor para el coeficiente alfa de Cronbach de0.908

El otro instrumento empleado ha sido la escala de Identificación Grupal (Tarrant, 2002) que incluye 13 ítems que evalúan la identificación que siente el sujeto con la organización en la cual trabaja actualmente. Consta de una escala de 11 puntos, siendo $0=$ completamente en desacuerdo hasta $10=$ completamente de acuerdo. ). La escala muestra un índice de consistencia interna de 0,415

\section{Procedimiento}

El criterio necesario para participar era ser mayor de edad y encontrarse activo laboralmente. Cada sujeto fue evaluado individualmente por uno de los investigadores. Para ello éstos se desplazaron hasta el domicilio de los participantes. Antes de la aplicación de los diferentes instrumentos cada uno de ellos expresó su deseo voluntario de participar en la investigación, e indico su pertenecía a una organización pública o privada.

Los cuestionarios fueron entregados a los sujetos, y los rellenaron delante de los investigadores preguntando todas las dudas que tuviesen con algún ítem en particular y resolviéndolas. El orden de realización de los dos cuestionarios se realizó de forma aleatoria, no siguiendo un orden preestablecido El intervalo de tiempo empleado fue de unos 10-15 minutos. Ambos cuestionarios se corrigieron según sus criterios establecidos y estandarizados de corrección mediante el programa estadístico SPSS.

Análisis

Para el análisis de los resultados se realizó el estudio descriptivo básico, comparación de medias a través de la prueba T-student, prueba de Levenne para igualdad de varianzas y Correlación de Pearson. 


\section{Resultados}

Tras la recogida de datos se llevó a cabo una serie de análisis estadísticos que describen la satisfacción laboral y la identidad organizacional de las empresas públicas y privadas a partir de los datos obtenidos en nuestra muestra. Los resultados obtenidos se muestran en la siguiente tabla (Tabla 1).

Tabla 1. Estadísticos descriptivos.

\begin{tabular}{lllll}
\hline & Empresa & N & Media & DT \\
\hline Satisfacción & Pública & 45 & 56,56 & 12,73 \\
laboral & Privada & 50 & 63,14 & 14,97 \\
\multirow{2}{\text{Identidad}}{$\begin{array}{l}\text { Organizacional } \\
\end{array}$} & Pública & 45 & 95,60 & 19,57 \\
& Privada & 50 & 99,28 & 22,96 \\
\hline
\end{tabular}

La correlación de las dos variables principales, satisfacción laboral e identificación organizacional, medida a través de la correlación de Pearson es significativa (Tabla 2). Además se ha realizado la prueba de homocedasticidad en las que se obtiene un $36 \%$ de varianza común.

Tabla 2. Correlaciones Satisfacción Laboral e Identidad Grupal

\begin{tabular}{lcc}
\hline & Satisfacción Laboral & Identidad Organizacional \\
\hline Satisfacción Laboral & --- & $.614^{* *}$ \\
Identidad Organizacional & $.614^{* *}$ & --- \\
\hline
\end{tabular}

Nota. ${ }^{* *} p<.01$

Posteriormente, se compararon las dos muestras (empresa pública/empresa privada) a través de la prueba T-student para muestras independientes. Se encontró que las empresas privadas presentan una mayor satisfacción que las públicas, (t93=-2`296, $p=.024)$.

En cuanto a la identificación organizacional no se encontró diferencias significativas en función de la muestra $\left(t_{93}=-.836, p=.405\right)$.

\section{Conclusión}

Esta investigación tenía como objetivo comparar las discrepancias entre el nivel de identificación y satisfacción en las empresas privadas y públicas. Tras la realización de la misma y una vez analizados los datos, los resultados obtenidos nos muestran que son varias las conclusiones a las que podemos llegar en relación con nuestras hipótesis.

Como predice el estudio, existe una correlación positiva entre la satisfacción laboral y la identificación organizacional. Estos resultados concuerdan con la revisión de la 
literatura especializada, concretamente con los estudios realizados por Mañas et al., (2007), los cuales consideraban que la satisfacción laboral es la principal variable interviniente en el compromiso o identidad organizacional y por Páez (2010), el cual defendía que una mayor involucración de los empleados en las decisiones de la empresa llevaría a una mayor identificación organizacional de los mismos, aumentando a su vez su satisfacción laboral.

Por otra parte, la satisfacción laboral es mayor en las empresas privadas que en las públicas. Esta satisfacción se consigue estableciendo un equilibrio entre el trabajo ideal y el actual. Esta diferencia de satisfacción de las empresas privadas frente a las públicas podría deberse al trato más familiar de las organizaciones privadas con el trabajador y a la posibilidad de ascenso y evolución dentro de la empresa, aspecto que es menos viable en las empresas públicas. Otro de los factores que pueden influir actualmente en estos resultados es la crisis económica. Los recortes del estado han afectado principalmente al sector público, reduciendo salarios y aumentando el horario laboral. Sin embargo, si una empresa privada no se ve afectada por la crisis, los trabajadores no tienen por qué sufrir las consecuencias de la misma. (González, J.S., 2013).

A razón de lo dicho anteriormente, estos resultados corroboran nuestras hipótesis principales a excepción de la referida a la mayor identificación organizacional en las empresas privadas, en comparación con las públicas. No se han encontrado diferencias significativas entre las empresas en relación a la identificación ya que las puntuaciones de cada sujeto muestran una desviación típica muy alejada de la media. Es decir, encontramos muchas diferencias individuales respecto a la variable identificación organizacional. Estas diferencias pueden deberse a que la identificación es una percepción subjetiva propia de cada sujeto, la cual pueden variar entre las personas de una misma empresa y depende de multitud de variables como puede ser la distintividad y prestigio del grupo, así como la identificación con los valores del mismo (Ashforth y Mael, 1989).

Una posible linea de investigación que podemos sugerir y que supera las limitaciones de este estudio, es investigar, con una muestra mayor la cual permita más generalización de los resultados, las razones de la mayor satisfacción laboral de las empresas públicas, de manera que estos factores puedan aplicarse a todos los ámbitos laborales y, así conseguir una mejor calidad de empleo de los trabajadores.

Por último sería interesante realizar un estudio longitudinal de las distintas empresas para analizar cuáles son las consecuencias de la crisis tanto para la satisfacción como para la identificación organizacional a lo largo de los años.

\section{Bibliografía}

Ashforth, B,y Mael, F. (1989). Social Identity theory and the organization. Academy of Management Review, 14(1), 20-39.

Cava, M.J.,Buelga, S., Herrero, J.,y Musitu, G. (2011). Estructura factorial de la adaptación española de la Escala de Identificación Grupal de Tarrant. Psicothema, 23, 772-777.

Díez, De castro, E.P., García del Junco, J., Martín Jiménez, F., y Periáñez Cristóbal, R. (2001). Administración y Dirección. Madrid: McGraw-Hill.

Gabriunas, P. (2010). La influencia del desempeño social corporativo en la satisfacción laboral de los empleados: una revisión teórica desde una perspectiva multinivel. Estudios Gerenciales, 26 (116). 
Henry, K.B., Arrow, H., y Carini, B. (1999). A tripartite model of group identification. Theory and Measurement. Small Group Research, 30(5), 558-581.

Hinknle, S., Taylor, L.A., Fox-Cardamone, D,L., y Crook, K.L. (1989) Intragroup identification and intragroup differentiation: A multicomponent approach. British Journal of Social Psychology, 28, 305-317.

Locke, E. (1976). The nature and causes of job satisfaction. En M. D. Dunnette (Ed.), Handbook of Industrial and Organizational Psychology (pp. 1297-1343). Chicago: Rand Mc Nally.

Mañas, M., Salvador, C., González, E., y Agulló, E. (2007). La satisfaccion y el bienestar psicológico como antecedentes del compromiso organizacional. Psicothema. Oviedo.

Maslow, A. (1943) A Theory of Human Motivation. Psychological Review, 50 (4).

Mottaz, C.J. ( 1988). "Work satisfaction among hospital nurses". Hospital and Health Services Administration, 33, 57-74.

Osca, A., Palací, F.J., Topa, G., Moriano, y J.A., Lisbona, A. (2012) Identificación de las organizaciones, actitudes y conductas en el trabajo. En Psicología de las organizaciones (pp. 163-205) Madrid: Sanz y Torres/UNED

Robbins, S. P., y Coulter, M. (2000). Administración. México: Pearson Education

Seashore, S. E., y Taber, T.D. (1975). Job satisfaction and their correlation. American Behavioral Scientist, 18, 333-386.

Tajfel, H. (1982). Social identity and intergroup relations. Cambridge: Cambridge University Press.

Turner, J.C. (1982). Towards a cognitive redefinition of the social group. En H. Tajfel (Ed), Social identity and intergroup relations (pp. 15-40.). Cambridge: Cambdirge University Press.

González, J.S., (2013). Hacienda volverá a congelar los sueldos a los empleados públicos en 2014 [elpaís.com] Recuperado de: http://economia.elpais.com/economia/2013/09/26/actualidad/1380209728_4432 25.html el 26 de septiembre de 2013

Van Knippenberg, D., y Van Schie, E.C. (2000). Foci and correlates of organizational identification. Journal of Occupational an Organizational Psychology, 73, 137147. 